\title{
Positivity of entropy production for the three-level maser
}

\author{
Alex Arash Sand Kalaee (1) and Andreas Wacker (1)* \\ Mathematical Physics and NanoLund, Lund University, 22100 Lund, Sweden
}

(Received 15 October 2020; accepted 21 December 2020; published 7 January 2021)

\begin{abstract}
Entropy production is a key concept of thermodynamics and allows one to analyze the operation of engines. For the Scovil-Schulz-DuBois heat engine, the archetypal three-level thermal maser coupled to thermal baths, it was argued that the common definition of heat flow may provide negative entropy production for certain parameters [Boukobza and Tannor, Phys. Rev. Lett. 98, 240601 (2007)]. Here, we show that this can be cured if corrections for detuning are properly applied to the energies used for the bath transitions. This method can be used more generally for the thermodynamical analysis of optical transitions where the modes of the light field are detuned from the transition energy.
\end{abstract}

DOI: 10.1103/PhysRevA.103.012202

\section{INTRODUCTION}

With the realization of masers and lasers quantum optics has proved fertile ground for thermodynamic research in open quantum systems. An archetypal example is the ScovilSchulz-DuBois heat engine based on a three-level maser driven by two heat baths of different temperatures [1]. This system served as a model to develop a variety of approaches for the microscopic description of quantum systems in contact with thermal baths in interaction with classical [2-6] or quantized [7-9] light fields.

Of key relevance is the formulation of work and heat in the quantum realm. References $[10,11]$ defined work flow (power) and heat flow by partitioning the time derivative of the expectation value of the full Hamiltonian, i.e., including the time-dependent interaction with classical degrees of freedom, such as a microwave field. Later, Boukobza and Tannor [5] proposed an alternative definition of power and heat flow by restricton to the bare Hamiltonian, which describes the system itself and lacks explicit time dependence. This is sometimes conceptually simpler and was, e.g., also used in Refs. [12,13]. The authors argued [14] that the bare heat flows always provide a positive entropy production [15] for the three-level maser, while this was not the case for full heat flows in their treatment, which thus may violate the second law of thermodynamics. (We use full and bare in the sense that they relate to the Hamiltonian from which the flows are derived.) The correct definition of heat and work is actually still an open issue; see, e.g., the discussion on page 339 of [16], where further references are given.

\footnotetext{
*Andreas.Wacker@fysik.lu.se
}

Published by the American Physical Society under the terms of the Creative Commons Attribution 4.0 International license. Further distribution of this work must maintain attribution to the author(s) and the published article's title, journal citation, and DOI. Funded by Bibsam.
In this work we study the definitions of work and heat for the three-level maser coupled to a classical microwave field, where the bath couplings are treated by a Lindblad dissipator as outlined in Sec. II. Section III focuses on the different definitions of heat and work, where we essentially follow Ref. [14], showing a violation of entropy production for the full approach. In Sec. IV we present a reformulation of their expressions, which allows for the correct identification of energies supplied by the baths. Using these we recover a strictly positive entropy production for the full heat flows.

\section{THE SYSTEM}

We consider the three-level system of Scovil and SchulzDuBois [1], consisting of an upper $(u)$ and lower $(l)$ maser level and the ground level $(g)$ (see Fig 1). Throughout this article we set $\hbar=k_{B}=1$ in order to simplify the notation. The full system Hamiltonian, $H=H_{0}+V(t)$, consists of the bare Hamiltonian $H_{0}=\omega_{u} \sigma_{u u}+\omega_{l} \sigma_{l l}$ and a modulating external field $V(t)=\epsilon\left(e^{i \omega_{d} t} \sigma_{l u}+e^{-i \omega_{d} t} \sigma_{u l}\right)$, where $\epsilon$ is the strength of the driving field, $\omega_{d}$ its modulating frequency, and we use the operators $\sigma_{i j}=|i\rangle\langle j|$. Without loss of generality the energy of the ground state $|g\rangle$ is set to 0 . The three-level system is connected to two bosonic reservoirs (baths), which are labeled by $\alpha$, where $\alpha \in\{u, l\}$. The bath $\alpha$ couples to the transition $|g\rangle \leftrightarrow|\alpha\rangle$ with strength $\gamma_{\alpha}$, where an average number of excitations $n_{\alpha}$ is available in the bath. The model and the analysis of its steady-state behavior summarized below follow recent work $[5,13,14]$.

The time evolution of the system density operator $\rho$ is assumed to be Markovian and governed by the Lindblad master equation [17]

$$
\dot{\rho}=-i[H(t), \rho]+\mathcal{L}_{u}[\rho]+\mathcal{L}_{l}[\rho],
$$

where the coupling to the baths is described by $\mathcal{L}_{\alpha}[\rho]=$ $\gamma_{\alpha} n_{\alpha} \mathcal{D}_{\sigma_{\alpha g}}[\rho]+\gamma_{\alpha}\left(n_{\alpha}+1\right) \mathcal{D}_{\sigma_{g \alpha}}[\rho]$ with the dissipator $\mathcal{D}_{\sigma}[\rho]=\sigma \rho \sigma^{\dagger}-\frac{1}{2}\left\{\sigma^{\dagger} \sigma \rho+\rho \sigma^{\dagger} \sigma\right\}$.

To simplify the master equation we remove the time dependence of the Hamiltonian by transforming the system to 


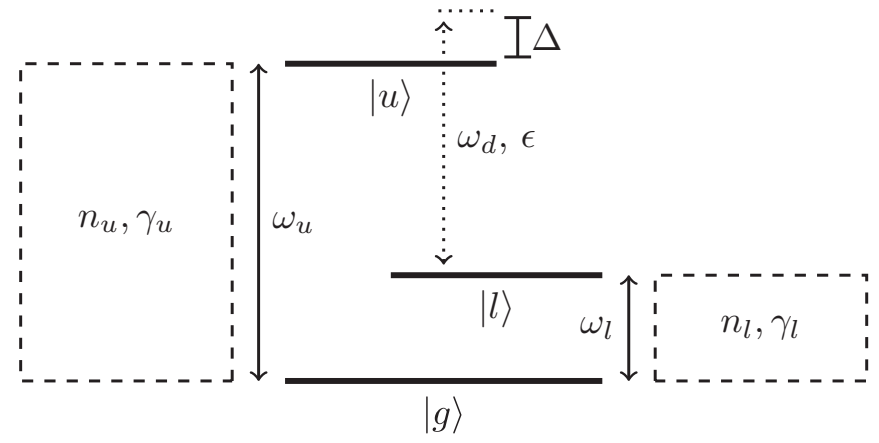

FIG. 1. Energy diagram of the three-level maser subjected to a modulating field (dotted arrow), where the transitions $g \leftrightarrow u$ and $g \leftrightarrow l$ (solid arrows) are coupled to different reservoirs. A finite value of $\Delta=\omega_{d}-\left(\omega_{u}-\omega_{l}\right)$ reflects the detuning between the modulating field and the energy level difference.

a rotating frame [13,14]. For $X=\omega_{l} \sigma_{l l}+\left(\omega_{l}+\omega_{d}\right) \sigma_{u u}$, we define $A^{\text {rot }}=U(t) A U^{\dagger}(t)$ according to the unitary operator $U(t)=e^{i X t}$. While the dissipative terms are unaffected by the choice of the rotating frame, the unitary part of the quantum evolution is determined by the Hamiltonian

$$
\widetilde{H}=H^{\mathrm{rot}}-X=-\Delta \sigma_{u u}+\epsilon\left(\sigma_{u l}+\sigma_{l u}\right)
$$

with the detuning parameter $\Delta=\omega_{d}-\left(\omega_{u}-\omega_{l}\right)$. Solving Eq. (1) for the steady state in the rotating frame (details are given in Appendix A) yields the net transition rate $R_{u \rightarrow l}$ from the upper to the lower level,

$$
R_{u \rightarrow l}=\frac{A\left(\gamma_{u}, \gamma_{l}, n_{u}, n_{l}, \epsilon\right)}{F\left(\gamma_{u}, \gamma_{l}, n_{u}, n_{l}, \epsilon, \Delta\right)}\left(n_{u}-n_{l}\right),
$$

where $A$ and $F$ are both positive [14] [see Eq. (A7)]. Thus $R_{u \rightarrow l}$ has the same sign as the difference $n_{u}-n_{l}$ between bath occupations which is driving the transitions.

\section{WORK, HEAT, AND ENTROPY}

Let the average energy in the system be $\langle E\rangle=\operatorname{Tr}\{\rho H\}$. The typical definitions of full power and full heat flows in the density matrix formalism are $[10,11]$

$$
P=\dot{W}=\operatorname{Tr}\{\rho \dot{H}\}, \quad \dot{Q}=\operatorname{Tr}\{\dot{\rho} H\},
$$

where we use the convention that positive values of $P$ and $\dot{Q}$ correspond to an increase in energy in the system.

Alternatively, some authors apply an alternative definition of the work and heat for systems coupled to a time-dependent external field which is based on the bare Hamiltonian, $\left\langle E_{0}\right\rangle=$ $\operatorname{Tr}\left\{\rho H_{0}\right\}[5,12]$. Based on the first law of thermodynamics the bare flows are identified from

$$
\dot{E}_{0}=-i \operatorname{Tr}\left\{\rho\left[H_{0}, V(t)\right]\right\}+\sum_{\alpha \in\{u, l\}} \operatorname{Tr}\left\{\mathcal{L}_{\alpha}[\rho] H_{0}\right\},
$$

where the first (unitary) term is interpreted as the bare power $P_{0}$ and the second (dissipative) term as the sum of bare heat flows $\dot{Q}_{0 \alpha}$ from the respective baths to the system. These terms can be evaluated in either the original or the rotating frame due to the invariance of the trace under cyclic permutations of operators. [This is simpler compared to the first definition with full power and heat flow Eq. (4), where the transformations $[\dot{A}]^{\text {rot }} \neq d A^{\text {rot }} / d t$ for $A=H, \rho$ are more involved.]

From these definitions the steady-state bare power and heat flow become (see Appendix B)

$$
\begin{aligned}
P_{0} & =-R_{u \rightarrow l}\left(\omega_{u}-\omega_{l}\right), \\
\dot{Q}_{0 u} & =+R_{u \rightarrow l} \omega_{u}, \\
\dot{Q}_{0 l} & =-R_{u \rightarrow l} \omega_{l} .
\end{aligned}
$$

We note that the bare power and heat flow correspond to the net transition rate $R_{u \rightarrow l}$ multiplied by the respective bare transition energies from $H_{0}$.

The second law of thermodynamics requires a positive definite entropy production. Spohn's entropy production function for the engine reads [15]

$$
\sigma=\frac{\partial S}{\partial t}-\frac{\dot{Q}_{u}}{T_{u}}-\frac{\dot{Q}_{l}}{T_{l}}
$$

where $S=\operatorname{Tr}\{\rho \ln \rho\}=\operatorname{Tr}\left\{\rho_{\text {steady state }}^{\text {rot }} \ln \rho_{\text {steady state }}^{\text {rot }}\right\}$ is the von Neumann entropy [18] of the three-level system, which is constant at steady state. The temperatures of the baths are commonly related to the mean occupations as

$$
T_{\alpha}=\frac{\omega_{\alpha}}{\ln \left(1+\frac{1}{n_{\alpha}}\right)}
$$

by using the appropriate Bose distribution function.

Using the bare heat flows from Eq. (6) Boukobza and Tannor found [14]

$$
\sigma_{0}=R_{u \rightarrow l}\left[\ln \left(1+\frac{1}{n_{l}}\right)-\ln \left(1+\frac{1}{n_{u}}\right)\right]>0,
$$

which is positive definite, as both factors have the same sign of $\left(n_{u}-n_{l}\right)$ [see Eq. (3)]. In contrast, using the full heat flows from Eq. (4) with temperatures from Eq. (8), Boukobza and Tannor [14] detected negative entropy production for some operation points. This suggested that the definition of work and heat based on the bare Hamiltonian, (5), should be preferred.

\section{RESOLUTION BY EFFECTIVE ENERGIES}

Here, we rewrite the results for the full power and heat flows evaluated from Eq. (4) in the form

$$
\begin{gathered}
P=-R_{u \rightarrow l} \omega_{d}, \\
\dot{Q}_{u}=+R_{u \rightarrow l} \tilde{\omega}_{u}, \\
\dot{Q}_{l}=-R_{u \rightarrow l} \tilde{\omega}_{l},
\end{gathered}
$$

with effective energies (see Appendix C)

$$
\begin{gathered}
\tilde{\omega}_{u}=\omega_{u}+\frac{\Delta \gamma_{u}\left(n_{u}+1\right)}{\gamma_{u}\left(n_{u}+1\right)+\gamma_{l}\left(n_{l}+1\right)}, \\
\tilde{\omega}_{l}=\omega_{l}-\frac{\Delta \gamma_{l}\left(n_{l}+1\right)}{\gamma_{u}\left(n_{u}+1\right)+\gamma_{l}\left(n_{l}+1\right)} .
\end{gathered}
$$

Comparing Eq. (6) with Eq. (10) we note that all flows are proportional to the transition rate $R_{u \rightarrow l}$, describing the roundtrip rate of the engine. However, there are different energy factors in each term. 
For vanishing detuning, $\Delta=0$, the respective energy factors in Eq. (6) and Eq. (10) agree. Here, the heat fluxes from the baths are determined by the level energies $\omega_{\alpha}$ and the power transferred from the light field is given by the photon energy $\omega_{d}$, as expected.

However, for finite detuning, i.e., $\Delta=\omega_{d}-\omega_{u}+\omega_{l} \neq 0$, energy conservation does not allow for this structure, where the full and the bare approach provide different remedies: In the bare approach based on Eq. (5), the power supplied from the ac field changes its energy factor $\omega_{d} \rightarrow \omega_{u}-\omega_{l}$ [see Eq. (6)]. This appears not to be physical, as a quantized ac field should have energies in portions of $\hbar \omega_{d}$, and thus may result in an error of the order $\Delta R_{u \rightarrow l}$ in the power. In contrast, for the full approach, the bare level energies are replaced by effective ones, $\omega_{\alpha} \rightarrow \tilde{\omega}_{\alpha}$ [see Eq. (10)], which satisfy $\omega_{d}=\tilde{\omega}_{u}-\tilde{\omega}_{l}$, so that energy conservation holds with the ac frequency of the field.

Here we argue that the effective energies, Eq. (11), should be taken seriously in the full approach and thus be used in the definitions of the bath temperatures

$$
\widetilde{T}_{\alpha}=\frac{\tilde{\omega}_{\alpha}}{\ln \left(1+\frac{1}{n_{\alpha}}\right)} .
$$

Then Eq. (7) provides

$$
\begin{aligned}
\sigma & =-\frac{\dot{Q}_{u}}{\widetilde{T}_{u}}-\frac{\dot{Q}_{l}}{\widetilde{T}_{l}}=R_{u \rightarrow l}\left[\frac{\tilde{\omega}_{l}}{\widetilde{T}_{l}}-\frac{\tilde{\omega}_{u}}{\widetilde{T}_{u}}\right] \\
& =R_{u \rightarrow l}\left[\ln \left(1+\frac{1}{n_{l}}\right)-\ln \left(1+\frac{1}{n_{u}}\right)\right],
\end{aligned}
$$

which is identical to the entropy production function, Eq. (9), from the bare approach and, most importantly, positive definite.

Now, we want to highlight the particular meaning of the energies $\tilde{\omega}_{u}, \tilde{\omega}_{l}$ from Eq. (11). Due to lifetime broadening the energies of levels $u$ and $l$ are smeared out by Lorentzian spectral functions (here normalized to 1 ),

$$
A_{\alpha}(\omega)=\frac{1}{2 \pi} \frac{\gamma_{\alpha}\left(1+n_{\alpha}\right)}{\left(\omega-\omega_{\alpha}\right)^{2}+\gamma_{\alpha}^{2}\left(1+n_{\alpha}\right)^{2} / 4},
$$

with a full width at half-maximum (FWHM) $\gamma_{\alpha}\left(1+n_{\alpha}\right)$ resulting from the decay of the states by relaxation to the ground level. This allows for energy-conserving transitions between level $u$ and level $l$ at the energy $\omega_{d}$ imposed by the ac field even if $\omega_{d} \neq \omega_{u}-\omega_{l}$ (see Fig. 2). Fermi's golden rule provides the transition rate from the initial level $u$ with the energy $\omega$ (similar but not necessarily equal to $\omega_{u}$ ):

$$
W_{u \rightarrow l}=2 \pi \epsilon^{2} A_{l}\left(\omega-\omega_{d}\right) \text {. }
$$

Weighting with the density $A_{u}(\omega)$ of the initial state and multiplying by the difference in occupation $f_{u}(\omega)-f_{l}\left(\omega-\omega_{d}\right)$ of the levels (technically, $f_{\alpha}$ is the ratio between the imaginary part of the lesser Green's function and the spectral function [19]), we obtain the net transition rate

$$
R_{u \rightarrow l}=2 \pi \epsilon^{2} \int d \omega A_{u}(\omega) A_{l}\left(\omega-\omega_{d}\right)\left[f_{u}(\omega)-f_{l}\left(\omega-\omega_{d}\right)\right] .
$$

Neglecting the energy dependence of $f_{\alpha}$ over the width of the spectral functions (which would be relevant to study disper-

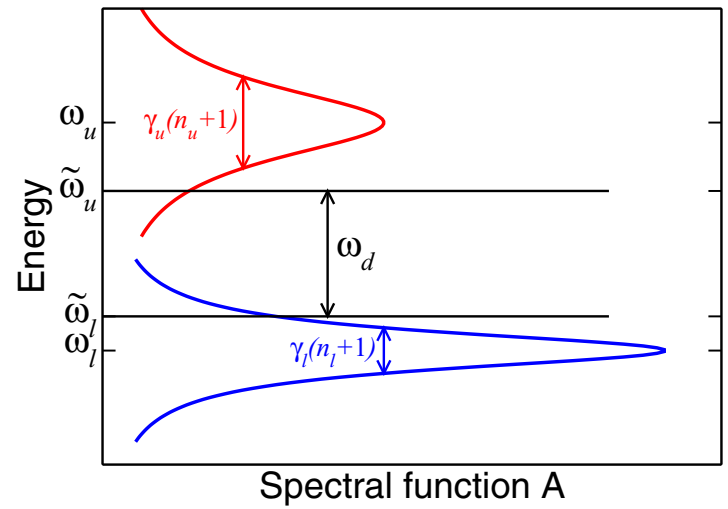

FIG. 2. Sketch of the spectral functions, (14), for the upper and lower levels, $u$ and $l$. The black arrow shows the optical transition at frequency $\omega_{d}$ not matching the energy difference of the bare states. The energies $\tilde{\omega}_{u}$ and $\tilde{\omega}_{l}$ are given by (11).

sive or Bloch gain [20]), we set $f_{u}(\omega)-f_{l}\left(\omega-\omega_{d}\right) \approx \rho_{u u}-$ $\rho_{l l}$. Then some algebra [see Eq. (D1)] results in expression (A6). This shows the equivalence of this treatment based on the Green's function with the density matrix calculations used above.

Equation (15) shows that there is not a single definite energy involved for the upper and lower levels, if broadening is taken into account. However, as the transitions occur with the weight $A_{u}(\omega) A_{l}\left(\omega-\omega_{d}\right)$, we can identify the average energy for the upper level involved in transitions

$$
\langle\omega\rangle_{u}=\frac{\int d \omega \omega A_{u}(\omega) A_{l}\left(\omega-\omega_{d}\right)}{\int d \omega A_{u}(\omega) A_{l}\left(\omega-\omega_{d}\right)}
$$

and obtain, after some algebra [see Eqs. (D1) and (D2)], $\langle\omega\rangle_{u}=\tilde{\omega}_{u}$ with $\tilde{\omega}_{u}$ from Eq. (11). The average energy for the lower level involved is then $\langle\omega\rangle_{l}=\langle\omega\rangle_{u}-\omega_{d}=\tilde{\omega}_{l}$. Thus we find that the effective levels from Eq. (11) are the average energies involved in the optical transition, if level broadening is taken into account. These are the average energies which need to be added to or removed from the respective bath after a transition has taken place in order to restore the previous state. Therefore the bath properties at these energies are of most relevance, which justifies the definition of temperature via Eq. (12).

Energy exchange with the bath $\alpha$ at energies different from $\omega_{\alpha}$ requires that the energies available in the baths cover a range of several $\gamma_{\alpha}$ 's around $\omega_{\alpha}$. In the Green's function picture, this is the basis for assuming an energy-independent self-energy (i.e., a constant width in the spectral function). For the Lindblad kinetics, the Markovian limit used requires a short bath correlation time $\tau_{B} \ll 1 / \gamma_{\alpha}$ [21] and, consequently, a spectral width of the bath well surpassing $\gamma_{\alpha}$. This demonstrates again the consistency between the Green's function-based treatment and the density matrix calculations.

Let us finally consider the Carnot efficiency of the engine. Reference [22] reported the occurrence of efficiencies above $1-T_{l} / T_{u}$ for $\Delta>0$ in the semiclassical treatment of the ac field. This is based on Eq. (15) in Ref. [22], which (in our 
notation) expresses the efficiency as

$$
\eta=\frac{-P}{\dot{Q}_{u}}=\frac{\omega_{d}}{\tilde{\omega}_{u}}=1-\frac{\tilde{\omega}_{l}}{\tilde{\omega}_{u}} .
$$

A positive power output $(-P)$ from the engine is based on $R_{u \rightarrow l}>0$ and thus requires $n_{u}>n_{l}$ by Eq. (3). Then our new definition of temperatures, (12), provides

$$
n_{u}>n_{l} \Rightarrow \frac{\tilde{T}_{u}}{\tilde{\omega}_{u}}>\frac{\tilde{T}_{l}}{\tilde{\omega}_{l}} \Leftrightarrow \frac{\tilde{\omega}_{l}}{\tilde{\omega}_{u}}>\frac{\tilde{T}_{l}}{\tilde{T}_{u}}
$$

so that Eq. (17) satisfies the Carnot efficiency $\eta<1-\tilde{T}_{l} / \tilde{T}_{u}$.

\section{CONCLUSION}

Both definitions of heat and work, applying either the full or the bare system Hamiltonian, provide identical (and positive definite) expressions for the entropy production for the common three-level maser driven by thermal baths. For the case of the full heat flow, it is crucial to carefully analyze the energies exchanged with the baths. These differ from the bare level energies if the ac field does not match the transition frequency. Disregarding this can provide violations of the second law as reported earlier [14]. While both the full and the bare approach are thermodynamically consistent and provide identical expressions for entropy production, the full approach requires an elaborate description of the energies transferred to the bath, which rely on the steady state in our treatment. Furthermore, it is an open issue whether this description can be extended to transient behavior, nonmonochromatic fields, or noncyclic operation [22]. On the other hand, the bare approach provides the transition frequency rather than the ac frequency in the work output, which provides a (typically small) error.

\section{ACKNOWLEDGMENTS}

We thank the Knut and Alice Wallenberg Foundation (project 2016.0089), the Swedish Research Council (project 2017-04287), and NanoLund for financial support.

\section{APPENDIX A: DETAILED DERIVATIONS OF THE STEADY-STATE SOLUTION}

After transforming to the rotating frame, Eq. (1) provides the equations of motion for $\rho_{i j}=\left\langle i\left|\rho^{\mathrm{rot}}\right| j\right\rangle$,

$$
\frac{d}{d t} \rho_{g g}=\gamma_{u}\left(n_{u}+1\right) \rho_{u u}+\gamma_{l}\left(n_{l}+1\right) \rho_{l l}-\left(n_{u} \gamma_{u}+n_{l} \gamma_{l}\right) \rho_{g g},
$$

$$
\begin{gathered}
\frac{d}{d t} \rho_{u u}=\gamma_{u} n_{u} \rho_{g g}-\gamma_{u}\left(n_{u}+1\right) \rho_{u u}+i \epsilon\left(\rho_{u l}-\rho_{u l}^{*}\right), \\
\frac{d}{d t} \rho_{l l}=\gamma_{l} n_{l} \rho_{g g}-\gamma_{l}\left(n_{l}+1\right) \rho_{l l}-i \epsilon\left(\rho_{u l}-\rho_{u l}^{*}\right), \\
\frac{d}{d t} \rho_{u l}=i \Delta \rho_{u l}+i \epsilon\left(\rho_{u u}-\rho_{l l}\right) \\
-\left[\gamma_{u}\left(n_{u}+1\right)+\gamma_{l}\left(n_{l}+1\right)\right] \rho_{u l} / 2 .
\end{gathered}
$$

At steady state (superscript "ss"), Eq. (A4) provides

$$
\rho_{u l}^{\mathrm{ss}}=\frac{-\epsilon\left(\rho_{u u}^{\mathrm{ss}}-\rho_{l l}^{\mathrm{ss}}\right)}{\Delta+i\left[\gamma_{u}\left(n_{u}+1\right)+\gamma_{l}\left(n_{l}+1\right)\right] / 2} .
$$

Furthermore, we identify the net rate of transitions between $u$ and $l$ due to the ac field:

$$
\begin{aligned}
R_{u \rightarrow l} & =-i \epsilon\left(\rho_{u l}^{\mathrm{ss}}-\rho_{u l}^{\mathrm{ss} *}\right)=C\left(\rho_{u u}^{\mathrm{ss}}-\rho_{l l}^{\mathrm{ss}}\right) \quad \text { with } \\
C & =\frac{\epsilon^{2}\left[\gamma_{u}\left(n_{u}+1\right)+\gamma_{l}\left(n_{l}+1\right)\right]}{\left[\gamma_{u}\left(n_{u}+1\right)+\gamma_{l}\left(n_{l}+1\right)\right]^{2} / 4+\Delta^{2}} .
\end{aligned}
$$

Using $\rho_{g g}=1-\rho_{u u}-\rho_{l l}$, Eqs. (A2), (A3), and (A6) provide the system of equations

$$
\begin{aligned}
\gamma_{u} n_{u} & =\left[\gamma_{u}\left(2 n_{u}+1\right)+C\right] \rho_{u u}^{\mathrm{ss}}+\left(\gamma_{u} n_{u}-C\right) \rho_{l l}^{\mathrm{ss}}, \\
\gamma_{l} n_{l} & =\left(\gamma_{l} n_{l}-C\right) \rho_{u u}^{\mathrm{ss}}+\left[\gamma_{l}\left(2 n_{l}+1\right)+C\right] \rho_{l l}^{\mathrm{ss}},
\end{aligned}
$$

with the solution

$$
\begin{aligned}
\rho_{u u}^{\mathrm{ss}} & =\frac{\gamma_{u} \gamma_{l} n_{u}\left(n_{l}+1\right)+C\left(\gamma_{u} n_{u}+\gamma_{l} n_{l}\right)}{\left[\gamma_{l}\left(2 n_{l}+1\right)+C\right]\left[\gamma_{u}\left(2 n_{u}+1\right)+C\right]-\left(\gamma_{l} n_{l}-C\right)\left(\gamma_{u} n_{u}-C\right)}, \\
\rho_{l l}^{\mathrm{ss}} & =\frac{\gamma_{u} \gamma_{l} n_{l}\left(n_{u}+1\right)+C\left(\gamma_{u} n_{u}+\gamma_{l} n_{l}\right)}{\left[\gamma_{l}\left(2 n_{l}+1\right)+C\right]\left[\gamma_{u}\left(2 n_{u}+1\right)+C\right]-\left(\gamma_{l} n_{l}-C\right)\left(\gamma_{u} n_{u}-C\right)},
\end{aligned}
$$

so that

$$
\rho_{u u}^{\mathrm{ss}}-\rho_{l l}^{\mathrm{ss}}=\frac{\gamma_{l} \gamma_{u}\left(n_{u}-n_{l}\right)}{\gamma_{u} \gamma_{l}\left(3 n_{u} n_{l}+2 n_{u}+2 n_{l}+1\right)+C\left[\gamma_{u}\left(3 n_{u}+1\right)+\gamma_{l}\left(3 n_{l}+1\right)\right]}
$$

is proportional to the occupation differences of the baths. Inserting this into Eq. (A6), we obtain Eq. (3) in the text, where

$$
\begin{aligned}
A= & \frac{\gamma_{l} \gamma_{u}}{4}\left[\gamma_{u}\left(n_{u}+1\right)+\gamma_{l}\left(n_{l}+1\right)\right] \epsilon^{2}, \\
F= & \frac{\gamma_{u}\left(n_{u}+1\right)+\gamma_{l}\left(n_{l}+1\right)}{2} \frac{\gamma_{u}\left(3 n_{u}+1\right)+\gamma_{l}\left(3 n_{l}+1\right)}{2} \epsilon^{2} \\
& +\frac{\gamma_{l} \gamma_{u}}{4}\left(3 n_{u} n_{l}+2 n_{u}+2 n_{l}+1\right)\left\{\frac{\left[\gamma_{u}\left(n_{u}+1\right)+\gamma_{l}\left(n_{l}+1\right)\right]^{2}}{4}+\Delta^{2}\right\}
\end{aligned}
$$

are quadratic polynomials in $\epsilon$. Thus the rate $R_{u \rightarrow l} \propto \epsilon^{2}$ for a small coupling $\epsilon$ to the ac field, while it saturates for $\epsilon^{2} \gtrsim$ $\gamma_{u}^{2}\left(n_{u}+1\right)^{2}+\gamma_{l}^{2}\left(n_{l}+1\right)^{2}+4 \Delta^{2} . A$ and $F$ are identical to the expressions in Eq. (13) in [14], where $\gamma_{0 \alpha}=\gamma_{\alpha} / 2$ is used. 


\section{APPENDIX B: HEAT AND WORK WITH THE BARE HAMILTONIAN}

The definition of heat flow from the bare Hamiltonian, (5), provides the bare heat entering from bath $u$ (note that the energy of the ground level is 0 ),

$$
\dot{Q}_{0 u}=\omega_{u}\left\langle u\left|\mathcal{L}_{u}(\rho)\right| u\right\rangle=\omega_{u}\left[\gamma_{u} n_{u} \rho_{g g}-\gamma_{u}\left(n_{u}+1\right) \rho_{u u}\right]
$$

Note that the diagonal elements of $\rho(t)$ are identical in the original and rotating frames. Thus, at steady state, Eq. (A2) provides $\dot{Q}_{0 u}^{\mathrm{ss}}=-i \omega_{u} \epsilon\left(\rho_{u l}^{\mathrm{ss}}-\rho_{l u}^{\mathrm{ss}}\right)=\omega_{u} R_{u \rightarrow l}$, and similarly we get $\dot{Q}_{0 l}^{\mathrm{ss}}=-\omega_{l} R_{u \rightarrow l}$. Finally, the bare work, (5), done by the field on our systems is

$$
\begin{aligned}
P_{0} & =i \operatorname{Tr}\left\{\rho\left[V(t), H_{0}\right]\right\}=i \operatorname{Tr}\left\{\rho^{\mathrm{rot}}\left[V^{\mathrm{rot}}, H_{0}^{\mathrm{rot}}\right]\right\} \\
& =i \epsilon\left(\omega_{u}-\omega_{l}\right)\left(\rho_{u l}-\rho_{u l}^{*}\right)
\end{aligned}
$$

which at steady state provides $P_{0}^{\mathrm{ss}}=-\left(\omega_{u}-\omega_{l}\right) R_{u \rightarrow l}$ so that $\dot{Q}_{0 u}^{\mathrm{ss}}+\dot{Q}_{0 l}^{\mathrm{ss}}+P_{0}^{\mathrm{ss}}=0$, as required by energy conservation. These are the terms provided in Eq. (6) without the superscript "ss."

\section{APPENDIX C: HEAT AND WORK WITH THE FULL HAMILTONIAN}

With definition (4), we obtain the power transferred to the system,

$$
\begin{aligned}
P(t) & =i \epsilon \omega_{d} \operatorname{Tr}\left\{\rho\left(|l\rangle\left\langle u\left|e^{i \omega_{d} t}-\right| u\right\rangle\langle l| e^{-i \omega_{d} t}\right)\right\} \\
& =i \epsilon \omega_{d}\left(\rho_{u l}-\rho_{l u}\right) \stackrel{\text { inss }}{=}-\omega_{d} R_{u \rightarrow l},
\end{aligned}
$$

which corresponds to the net rate of absorbed photons $\left(-R_{u \rightarrow l}\right)$ times the photon energy $\omega_{d}$. [Note that we have defined $\rho_{u l}$ in the rotating frame (see Appendix A), so that $\rho_{u l}=\left\langle u\left|\rho^{\mathrm{rot}}\right| l\right\rangle=\langle u|\rho| l\rangle e^{i \omega_{d} t}$.]

For the heat flow, the unitary evolution of $\rho(t)$ due to the Hamiltonian does not contribute, as $\operatorname{Tr}\{[\rho, H] H\}=$ $\operatorname{Tr}\{\rho[H, H]\}=0$, where we have used the invariance of the trace under cyclic permutations. Thus we can restrict ourselves to the nonunitarian part here. Then the part with $H_{0}$ provides the heat current $\dot{Q}_{0 u}$ from Eq. (B1). We have to add the part with $V(t)$ and find

$$
\begin{aligned}
\dot{Q}_{u} & =\dot{Q}_{0 u}+\epsilon\left\langle u\left|\mathcal{L}_{u}[\rho]\right| l\right\rangle e^{i \omega_{d} t}+\left\langle l\left|\mathcal{L}_{u}[\rho]\right| u\right\rangle e^{-i \omega_{d} t} \\
& =\dot{Q}_{0 u}-\epsilon \frac{\gamma_{u}\left(n_{u}+1\right)}{2}\left(\rho_{u l}+\rho_{l u}\right)
\end{aligned}
$$

Using Eqs. (A5) and (A6) we get at steady state

$$
\begin{gathered}
\dot{Q}_{u}^{\mathrm{ss}}=\dot{Q}_{0 u}^{\mathrm{ss}}-\frac{\gamma_{u}\left(n_{u}+1\right)}{2} \frac{\operatorname{Re}\left\{\rho_{u l}^{\mathrm{ss}}\right\}}{\operatorname{Im}\left\{\rho_{u l}^{\mathrm{ss}}\right\}} R_{u \rightarrow l}=R_{u \rightarrow l} \tilde{\omega}_{u}, \\
\dot{Q}_{l}^{\mathrm{ss}}=\dot{Q}_{0 l}^{\mathrm{ss}}-\frac{\gamma_{l}\left(n_{l}+1\right)}{2} \frac{\operatorname{Re}\left\{\rho_{u l}^{\mathrm{ss}}\right\}}{\operatorname{Im}\left\{\rho_{u l}^{\mathrm{ss}}\right\}} R_{u \rightarrow l}=-R_{u \rightarrow l} \tilde{\omega}_{l},
\end{gathered}
$$

with

$$
\begin{aligned}
& \tilde{\omega}_{u}=\omega_{u}+\frac{\Delta \gamma_{u}\left(n_{u}+1\right)}{\gamma_{u}\left(n_{u}+1\right)+\gamma_{l}\left(n_{l}+1\right)}, \\
& \tilde{\omega}_{l}=\omega_{l}-\frac{\Delta \gamma_{l}\left(n_{l}+1\right)}{\gamma_{u}\left(n_{u}+1\right)+\gamma_{l}\left(n_{l}+1\right)},
\end{aligned}
$$

where $\operatorname{Re}\{z\}$ and $\operatorname{Im}\{z\}$ denote, respectively, the real and imaginary parts of a complex value $z$. The full power and heat flow satisfy energy conservation, $P^{\mathrm{ss}}+\dot{Q}_{u}^{\mathrm{ss}}+\dot{Q}_{l}^{\mathrm{ss}}=0$, and provide Eqs. (10) and (11), where we have omitted the superscript "ss."

\section{APPENDIX D: CONVOLUTION OF LORENTZIANS}

We consider the function

$$
P(\omega, \Delta)=\frac{1}{2 \pi} \frac{2 \gamma_{u}}{\omega^{2}+\gamma_{u}^{2}} \frac{2 \gamma_{l}}{(\omega-\Delta)^{2}+\gamma_{l}^{2}},
$$

which is the product of two spectral functions with FWHM $2 \gamma_{\alpha}$. Then with the residue theorem we find

$$
\begin{aligned}
\int d \omega P(\omega, \Delta) & =i\left[\frac{2 \gamma_{u}}{2 i \gamma_{u}} \frac{2 \gamma_{l}}{\left(i \gamma_{u}-\Delta\right)^{2}+\gamma_{l}^{2}}+\frac{2 \gamma_{u}}{\left(\Delta+i \gamma_{l}\right)^{2}+\gamma_{u}^{2}} \frac{2 \gamma_{l}}{2 i \gamma_{l}}\right] \\
& =\frac{2 \gamma_{l}\left[\left(\Delta+i \gamma_{l}\right)^{2}+\gamma_{u}^{2}\right]+2 \gamma_{u}\left[\left(\Delta-i \gamma_{u}\right)^{2}+\gamma_{l}^{2}\right]}{\left[\left(\Delta-i \gamma_{u}\right)^{2}+\gamma_{l}^{2}\right]\left[\left(\Delta+i \gamma_{l}\right)^{2}+\gamma_{u}^{2}\right]} \\
& =\frac{2\left(\gamma_{u}+\gamma_{l}\right)\left[\Delta^{2}-2 i \Delta\left(\gamma_{u}-\gamma_{l}\right)-\left(\gamma_{u}-\gamma_{l}\right)^{2}\right]}{\left[\Delta^{2}+\left(\gamma_{u}+\gamma_{l}\right)^{2}\right]\left[\Delta^{2}-2 i \Delta\left(\gamma_{u}-\gamma_{l}\right)-\left(\gamma_{u}-\gamma_{l}\right)^{2}\right]}=\frac{2\left(\gamma_{u}+\gamma_{l}\right)}{\Delta^{2}+\left(\gamma_{u}+\gamma_{l}\right)^{2}},
\end{aligned}
$$

where the third identity is verified by comparing the results of the products in the numerator and denominator, respectively. The main result is that we obtain a Lorentzian with the sum of the individual widths. Similarly we find

$$
\begin{aligned}
\int d \omega \omega P(\omega, \Delta) & =i\left[\frac{2 \gamma_{u}}{2 i \gamma_{u}} \frac{i \gamma_{u} 2 \gamma_{l}}{\left(i \gamma_{u}-\Delta\right)^{2}+\gamma_{l}^{2}}+\frac{2 \gamma_{u}\left(\Delta+i \gamma_{l}\right)}{\left(\Delta+i \gamma_{l}\right)^{2}+\gamma_{u}^{2}} \frac{2 \gamma_{l}}{2 i \gamma_{l}}\right] \\
& =\frac{2 i \gamma_{u} \gamma_{l}\left[\left(\Delta+i \gamma_{l}\right)^{2}+\gamma_{u}^{2}\right]+2 \gamma_{u}\left(\Delta+i \gamma_{l}\right)\left[\left(\Delta-i \gamma_{u}\right)^{2}+\gamma_{l}^{2}\right]}{\left[\left(\Delta-i \gamma_{u}\right)^{2}+\gamma_{l}^{2}\right]\left[\left(\Delta+i \gamma_{l}\right)^{2}+\gamma_{u}^{2}\right]} \\
& =\frac{2 \gamma_{u} \Delta\left[\Delta^{2}-2 i \Delta\left(\gamma_{u}-\gamma_{l}\right)-\left(\gamma_{u}-\gamma_{l}\right)^{2}\right]}{\left[\Delta^{2}+\left(\gamma_{u}+\gamma_{l}\right)^{2}\right]\left[\Delta^{2}-2 i \Delta\left(\gamma_{u}-\gamma_{l}\right)-\left(\gamma_{u}-\gamma_{l}\right)^{2}\right]}=\frac{2 \gamma_{u} \Delta}{\Delta^{2}+\left(\gamma_{u}+\gamma_{l}\right)^{2}} .
\end{aligned}
$$


[1] H. E. Scovil and E. O. Schulz-DuBois, Three-Level Masers as Heat Engines, Phys. Rev. Lett. 2, 262 (1959).

[2] W. E. Lamb, Jr., Theory of an optical maser, Phys. Rev. 134, A1429 (1964).

[3] E. Geva and R. Kosloff, Three-level quantum amplifier as a heat engine: A study in finite-time thermodynamics, Phys. Rev. E 49, 3903 (1994).

[4] E. Geva and R. Kosloff, The quantum heat engine and heat pump: An irreversible thermodynamic analysis of the threelevel amplifier, J. Chem. Phys. 104, 7681 (1996).

[5] E. Boukobza and D. J. Tannor, Thermodynamics of bipartite systems: Application to light-matter interactions, Phys. Rev. A 74, 063823 (2006).

[6] M. O. Scully, K. R. Chapin, K. E. Dorfman, M. B. Kim, and A. Svidzinsky, Quantum heat engine power can be increased by noise-induced coherence, Proc. Natl. Acad. Sci. USA 108, 15097 (2011).

[7] E. Boukobza and D. J. Tannor, Thermodynamic analysis of quantum light amplification, Phys. Rev. A 74, 063822 (2006).

[8] A. Ghosh, D. Gelbwaser-Klimovsky, W. Niedenzu, A. I. Lvovsky, I. Mazets, M. O. Scully, and G. Kurizki, Two-level masers as heat-to-work converters, Proc. Natl. Acad. Sci. USA 115, 9941 (2018).

[9] W. Niedenzu, M. Huber, and E. Boukobza, Concepts of work in autonomous quantum heat engines, Quantum 3, 195 (2019).

[10] W. Pusz and S. L. Woronowicz, Passive states and KMS states for general quantum systems, Commun. Math. Phys. 58, 273 (1978).

[11] R. Alicki, The quantum open system as a model of the heat engine, J. Phys. A 12, L103 (1979).
[12] G. Kiršanskas, M. Franckié, and A. Wacker, Phenomenological position and energy resolving Lindblad approach to quantum kinetics, Phys. Rev. B 97, 035432 (2018).

[13] N. Jaseem, M. Hajdušek, V. Vedral, R. Fazio, L.-C. Kwek, and $\mathrm{S}$. Vinjanampathy, Quantum synchronization in nanoscale heat engines, Phys. Rev. E 101, 020201(R) (2020).

[14] E. Boukobza and D. J. Tannor, Three-Level Systems as Amplifiers and Attenuators: A Thermodynamic Analysis, Phys. Rev. Lett. 98, 240601 (2007).

[15] H. Spohn, Entropy production for quantum dynamical semigroups, J. Math. Phys. 19, 1227 (1978).

[16] D. Gelbwaser-Klimovsky, W. Niedenzu, and G. Kurizki, Thermodynamics of quantum systems under dynamical control, in Advances In Atomic, Molecular, and Optical Physics, edited by E. Arimondo, C. C. Lin, and S. F. Yelin (Academic Press, San Diego, CA, 2015), Vol. 64, pp. 329-407.

[17] G. Lindblad, On the generators of quantum dynamical semigroups, Commun. Math. Phys. 48, 119 (1976).

[18] J. von Neumann, Mathematische Grundlagen der Quantenmechanik (Springer, Berlin, 1932).

[19] H. Haug and A.-P. Jauho, Quantum Kinetics in Transport and Optics of Semiconductors (Springer, Berlin, 1996).

[20] A. Wacker, Lasers: Coexistence of gain and absorption, Nat. Phys. 3, 298 (2007).

[21] H.-P. Breuer and F. Petruccione, Open Quantum Systems (Oxford University Press, Oxford, UK, 2006).

[22] E. Boukobza and H. Ritsch, Breaking the Carnot limit without violating the second law: A thermodynamic analysis of offresonant quantum light generation, Phys. Rev. A 87, 063845 (2013). 